\title{
CIAS detection of Fasciola hepatica/F. gigantica intermediate forms in bovines from Bangladesh
}

\section{Syed Ali Ahasan ${ }^{1,2}$, M. Adela Valero ${ }^{2 *}$, Emdadul Haque Chowdhury ${ }^{1}$, Mohammad Taohidul Islam ${ }^{3}$, Mohammad Rafiqul Islam', Mohammad Motahar Hussain Mondal'4, Raquel V. Peixoto², Lavinia Berinde ${ }^{2,5}$, Miroslava Panova² and Santiago Mas-Coma ${ }^{2}$}

\begin{abstract}
${ }^{1}$ Department of Pathology, Faculty of Veterinary Science, Bangladesh Agricultural University, Mymensingh 2202, Bangladesh; ${ }^{2}$ Departamento de Parasitología, Facultad de Farmacia, Universidad de Valencia, Av. Vicente Andrés Estellés s/n, 46100 Burjassot, Valencia, Spain; ${ }^{3}$ Department of Medicine, Faculty of Veterinary Science, Bangladesh Agricultural University, Mymensingh 2202, Bangladesh; ${ }^{4}$ Department of Parasitology, Faculty of Veterinary Science, Bangladesh Agricultural University, Mymensingh 2202 , Bangladesh; ${ }^{5}$ Department of Microbiology, University of Medicine and Pharmacy "Iuliu Hatieganu",

Louis Pasteur street No. 6, Cluj-Napoca 400394, Romania
\end{abstract}

\begin{abstract}
Fascioliasis is an important food-borne parasitic zoonosis caused by two trematode species, Fasciola hepatica and Fasciola gigantica. The characterisation and differentiation of Fasciola populations is crucial to control the disease, given the different transmission, epidemiology and pathology characteristics of the two species. Lineal biometric features of adult liver flukes infecting livestock have been studied to characterise and discriminate fasciolids from Bangladesh. An accurate analysis was conducted to phenotypically discriminate between fasciolids from naturally infected bovines (cattle, buffaloes) throughout the country. Morphometric analyses were made with a computer image analysis system (CIAS) applied on the basis of standardised measurements and the logistic model of the body growth and development of fasciolids in the different host groups. Since it is the first ever comprehensive study of this kind undertaken in Bangladesh, the results are compared to pure fasciolid populations of $F$. hepatica from the European Mediterranean area and F. gigantica from Burkina Faso, geographical areas where both species do not co-exist. Principal component analysis showed that the biometric characteristics of fasciolids from Bangladesh are situated between $F$. hepatica and F. gigantica standard populations, indicating the presence of phenotypes of intermediate forms in Bangladesh. These results are analysed by considering the present emergence of animal fascioliasis, the local lymnaeid fauna, the impact of climate change, and the risk of human infection in the country.
\end{abstract}

\section{Keywords}

Fasciola hepatica, Fasciola gigantica, forms, multivariate analysis, CIAS, Bangladesh.

\section{Introduction}

Fascioliasis, a food-borne zoonosis caused by liver flukes of the genus Fasciola (Trematoda: Digenea), is a serious animal health problem worldwide and is considered the most important helminth infection in ruminants causing considerable socioeconomic problems in tropical countries (Mas-Coma et al. 2009a). This disease in ruminants causes substantial economic losses to rural agricultural communities and commercial animal producers due to the death of infected animals, condemnation of affected livers and production losses associated with reduced feed conversion efficiency worldwide (Torgerson and Claxton 1999; Spithill et al. 1999).
Additionally, studies performed in recent years have shown it to be an important public health problem as well. Human cases have been increasing in the five continents, including estimates of human infection of up to 17 million people, or even higher depending from the hitherto unknown situations in Asia and Africa (Mas-Coma et al. 2009a). This disease has recently proven to have a great morbidity impact throughout the biliary or chronic period of the disease (Valero et al. 2003, 2006a, 2008; Girones et al. 2007), and not only due to the pathogenicity of the acute phase as hitherto believed (Chen and Mott, 1990). Clinical studies have shown this disease to be pronouncedly complex, giving rise to progressive general deterioration of the patients, with sequelae sometimes 
leaving them handicapped and frail, even including fatal cases (Mas-Coma et al. 2014a). The diagnosis of the liver fluke infection in humans poses, moreover, many problems related to the different epidemiological scenarios and transmission patterns, as well as to the different situations in individual patients and due to the different periods this disease shows in humans (Valero et al. 2009a; Mas-Coma et al. 2014b). Additionally, recent epidemiological studies have proven it to be related to climate and global changes (Mas-Coma et al. 2008, 2009b; Afshan et al. 2014), as a consequence of the pronounced dependence of the fluke life cycle stages regarding the weather and other abiotic factors of the environment (Fuentes et al. 1999, 2001). The World Health Organization (WHO) has recognized fascioliasis as an emerging human disease, with human infection reported in more than 71 countries and 180 million people presently at risk of infection (WHO, 2013).

The two causal agents of this disease, Fasciola hepatica and $F$. gigantica, are two closely related species. The definitive host range of both fasciolid species is very large, including herbivorous mammals, mainly livestock species, while freshwater snails of the family Lymnaeidae act as intermediate hosts or vectors (Bargues and Mas-Coma, 2005). Whereas F. gigantica occurs mainly in tropical areas of Africa and Asia, $F$. hepatica prefers temperate and cooler areas throughout the five continents, and both species overlap in subtropical zones of Africa and Asia (Mas-Coma et al. 2009a). Intermediate forms appearing in overlap zones of southern Asia and Southeast Asia pose problems in the identification of the flukes involved in the infection of both humans and animals (Mas-Coma et al. 2009a; Ichikawa et al. 2011).

Despite the importance of distinguishing between infections by one or another fasciolid species, due to their different transmission, epidemiology, pathology and control characteristics, there is, unfortunately, neither a direct coproantigen test nor an indirect immunological test available for their differential diagnosis (Valero et al. 2009a, b, 2012a, b; Mas-Coma et al. 2014b). Diagnostic tools available at present are only useful to differentiate fascioliasis from other diseases. Hitherto, specific differentiation can only be made by either a phenotypic study of adult flukes by means of morphometric tools (Periago et al. 2006; Valero et al. 2009a, 2012c; Ashrafi et al. 2006, 2015; Asfhan et al. 2013) or genotypically by molecular tools (Mas-Coma et al. 2009a).

The overlapping distribution of $F$. hepatica and F. gigantica has also led to a long-lasting controversy concerning the taxonomic identity of the Fasciola species found in countries of the Far East, in which some resemble $F$. hepatica, whereas others resemble $F$. gigantica, with intermediate forms also being present and involving phenomena such as abnormal gametogenesis, diploidy, triploidy and mixoploidy, parthenogenesis and hybridisation events between different genotypes (Mas-Coma et al. 2009a). A comprehensive multidisciplinary study has, however, demonstrated that $F$. hepatica and $F$. gigantica should be considered valid species, despite their ca- pacity to hybridise and give rise to intermediate forms in overlapping areas (Mas-Coma et al. 2009a).

Bangladesh is one of the Asian countries where fascioliasis is the most prevalent parasitic disease in cattle, buffaloes, goats and sheep (Nooruddin and Islam, 1996; Hossain et al. 2011). An earlier survey revealed that $71.55 \%$ of cattle, $14.6 \%$ of sheep and goats and $100 \%$ of buffaloes are affected by the liver-fluke (Bhuiyan, 1970). A more recent report (BASUSDA, 2012) showed that $10.42 \%$ cattle, $9 \%$ goats and $36.61 \%$ of buffaloes are infected with Fasciola among snail borne trematode infections in Bangladesh. Recent postmortem examinations revealed a Fasciola infection rate of $35.27 \%$ irrespective of geographical areas and host animal species (unpublished data, 2013).

Unfortunately, appropriate phenotypic analyses to assess the presence and phenotypic features of the fasciolids have never been carried out in Bangladesh. The present study represents the first attempt within that endeavour. Bovines, as the most widespread livestock species in the country, were been the hosts selected. An approach by means of a computer image analysis system (CIAS) (Valero et al. 2005, 2012c) was applied for the morphometric characterisation of the liver flukes.

\section{Materials and Methods}

\section{Geographic location, collection and examination of flukes}

Bangladesh is a low-lying, riverine country located in south Asia with a vast marshy jungle coastline of $710 \mathrm{~km}$ on the northern littoral of the Bay of Bengal threatened by floods and droughts. It has a tropical monsoon climate characterised by heavy seasonal rainfall, high temperatures, and high humidity. The country has an area of 147,570 square kilometers and is bordered on the west, north, and east by a 4,095-km land frontier with India and, in the southeast, by a short land and water frontier $(193 \mathrm{~km})$ with Burma (Myanmar). In the south, there is a highly irregular deltaic coastline of about $580 \mathrm{~km}$, fissured by many rivers and streams flowing into the Bay of Bengal. Bangladesh comprises mostly irrigated fields which provide excellent breeding grounds for the development and survival of freshwater snails serving as potential intermediate hosts for a variety of digenetic trematode parasites.

Adult fasciolids were collected directly from the livers of naturally infected Nili-Ravi and Murrah type domestic water buffalo (Bubalus bubalis) $(\mathrm{n}=51)$ and Indian zebu (Bos indicus) $(\mathrm{n}=51)$, at necropsy from different locations in four different broad agro-ecological zones of Bangladesh between April and November 2012 (Table I and Fig. 1) with the help of rubber-coated forceps in order to avoid any structural damage to the flukes. Fasciolid specimens providing the largest worm variability in their size, maturity and gravid uteri were used for characterisation. Individual worms were washed extensively in physiological saline $(0.85 \% \mathrm{NaCl})$ to remove blood and bile. The living flukes were washed with and incu- 
Table I. Breeding sites of the bovine definitive hosts (with latitude, longitude and altitude) and traditional agro-ecological zones in Bangladesh where the liver-fluke samples were collected. Location on map (Fig. 1) indicated by letters

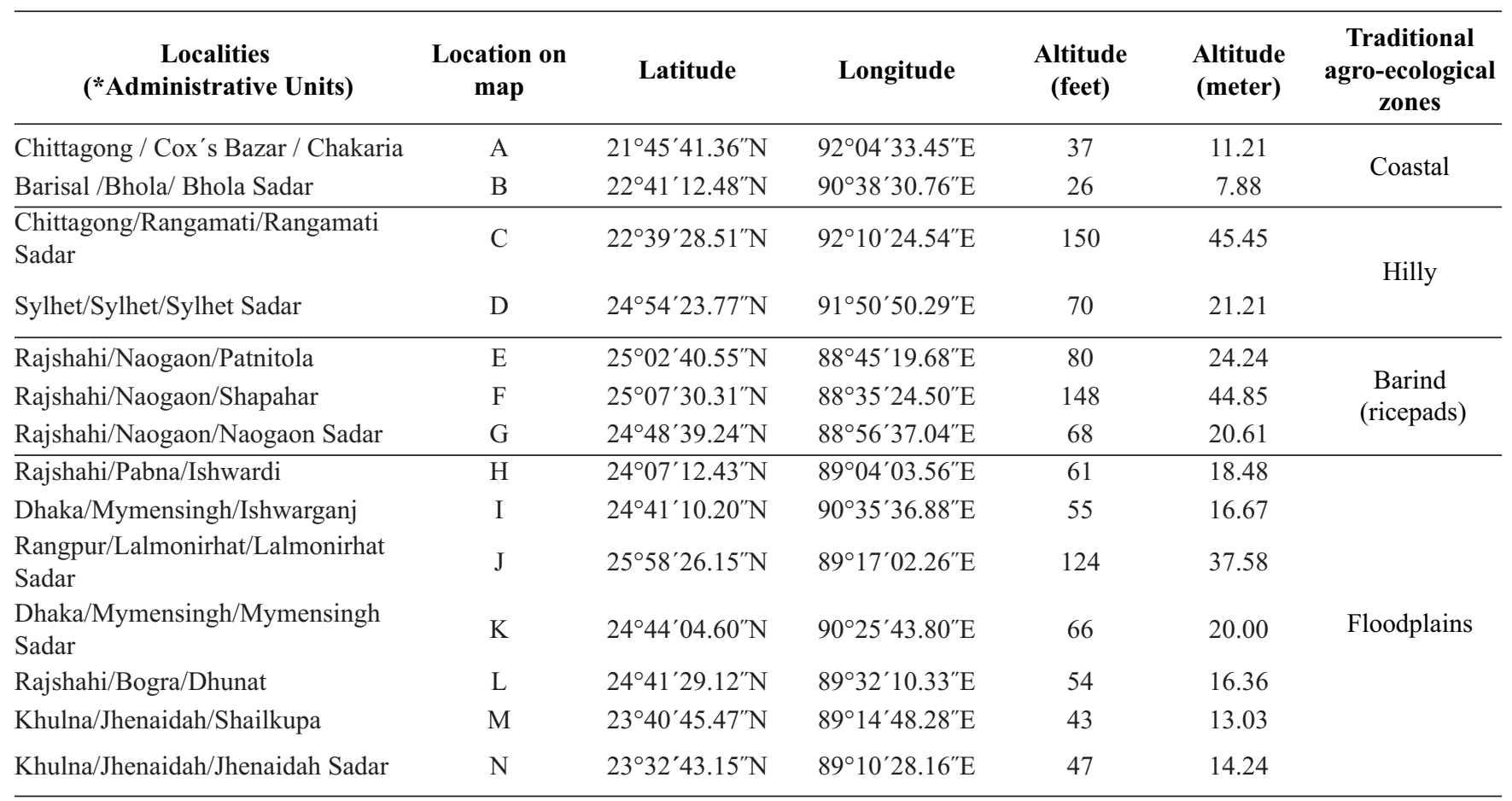

*Administrative units of Division / District / Upazila

bated in PBS at $37^{\circ} \mathrm{C}$ for a short while to allow them to expel the gut contents. The flukes were afterwards placed between two microscopic slides and adult worms were observed using a stereomicroscope to verify the presence of eggs in the uterus.

Fasciolid specimens were fixed in AFA solution $(95 \%$ alcohol -20 parts, formalin -6 parts, glacial acetic acid - 1 part, distilled water -40 parts) between two slides with little pressure, depending on the thickness of the flukes. The flukes were stained with Semichon's carmine solution (glacial acetic acid and distilled water by equal amount, saturation by dissolving carmine powder heated at $95-100{ }^{\circ} \mathrm{C}$ for 15 minutes and on filtration dilute with the same amount of $70 \%$ alcohol as stock solution) and subsequently differentiated, dehydrated and mounted with DPX.

\section{Morphometrics}

The morphometric study was conducted to phenotypically discriminate adult flukes from the naturally infected ruminants. Studies were performed using a computer image analysis system (CIAS) on the basis of standardised measurements known to be useful for the differentiation of fasciolid species (Valero et al. 2005, 2009a; Periago et al. 2006, 2008; Afshan et al. 2013). Standardised measurements were taken using a microscope and images captured by a digital camera (Nikon Coolpix), which were then analysed by image analysis soft- ware (ImagePro plus version 5.0 for Windows, Media Cybernetics, Silver Spring, Maryland, USA). Since it is the first time that such a study has been performed in Bangladesh, the results are compared to pure fasciolid populations also from boivines, namely: (i) F. hepatica from Valencia, Spain and Corsica, France; and (ii) F. gigantica from Burkina Faso. These pure fasciolid geographical origins are areas where both species do not co-exist (Periago et al. 2006). All the previously published data on $F$. hepatica from Spain (Europe) and F. gigantica from Burkina Faso (Africa) (Periago et al. 2006) are used as a standard references.

The following standardized measurements were taken (Fig. 2):

- Lineal biometric characters $(\mathrm{mm})$ : body length (BL), maximum body width (BW), body perimeter (BP), body roundness (BR), distance between the oral sucker and ventral sucker (OS-VS), distance between the ventral sucker and the union of the vitelline glands (VS-Vit), distance between the union of the vitelline glands and the posterior end of the body (Vit-P), distance between the ventral sucker and the posterior end of the body (VS-P), pharynx length (PL), pharynx width (PW).

- Areas $\left(\mathrm{mm}^{2}\right)$ : body area (BA).

- Ratios: BL over BW (BL/BW), BL over the distance between the VS and P (BL/VS-P), PL over PW (PL/PW), BP over BL (BP/BL), BL over distance between VS and Vit 


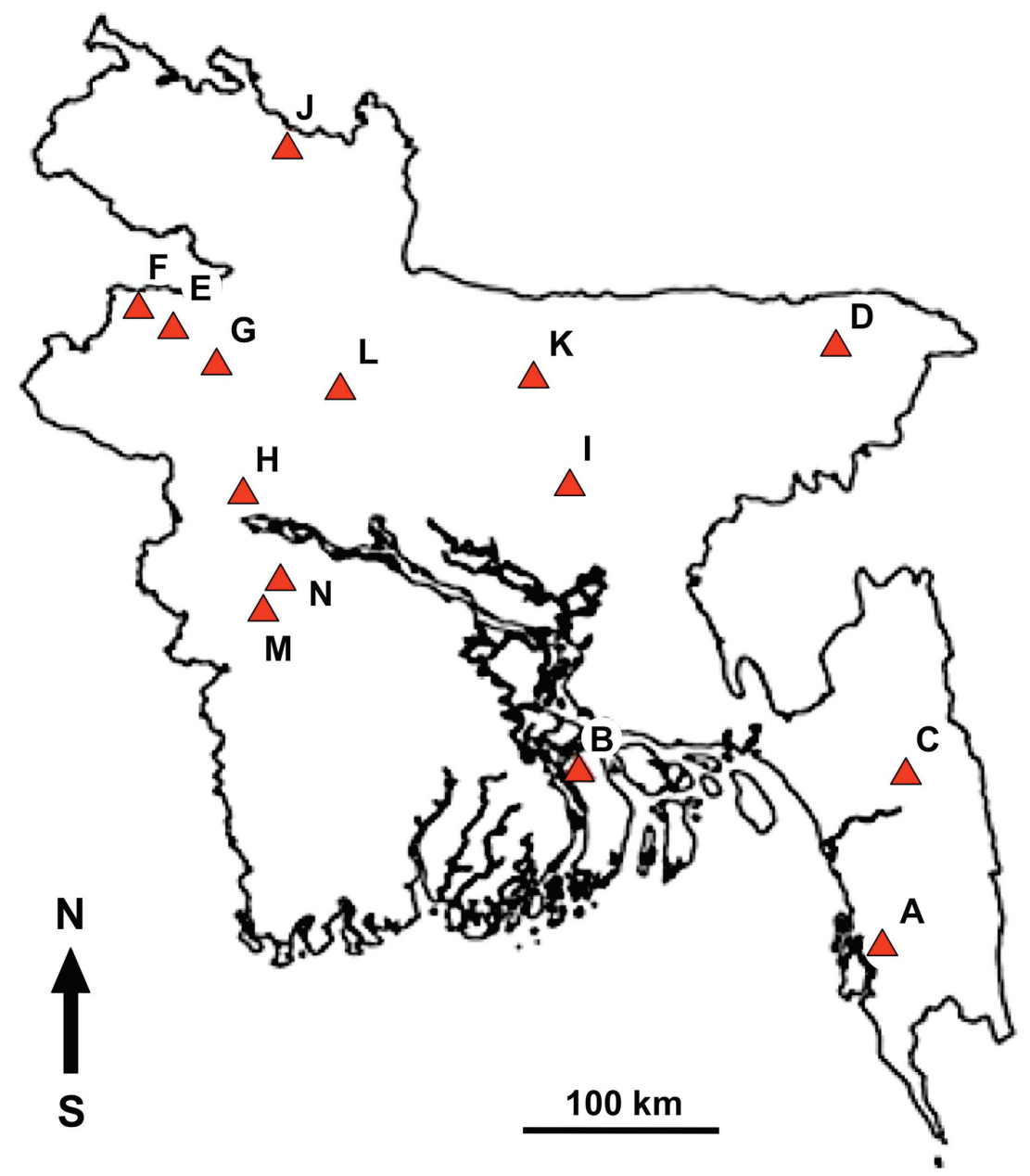

Fig. 1. Geographic map of Bangladesh, showing the sites where the hosts of the liver flukes analysed were bred. GIS data of each site are indicated in Table I. A - Chakaria; B - Bhola Sadar; C - Rangamati Sadar; D - Sylhet Sadar; E - Patnitola; F - Shapahar; G - Naogaon Sadar; H - Ishwardi; I - Ishwarganj; J - Lalmonirhat Sadar; K - Mymensingh Sadar; L - Dhunat; M - Shailkupa; N - Jhenaidah Sadar

(BL/VS-Vit) and $\mathrm{BL}$ over distance between Vit and $\mathrm{P}$ (BL/Vit-P); BA over BL (BA/BL), BW (BA/BW), BP (BA/BP), distance between VS and Vit (BA/VS-Vit), distance between Vit and P (BA/Vit-P) and distance between VS and P (BA/VS-P).

- Body roundness $(\mathrm{BR}=\mathrm{BP} 2 / 4 \pi \mathrm{BA})$ was used to measure the body shape. A circular object will always have a roundness of 1.00, while more irregular objects will have larger values, indicating how circular an object is (Periago et al. 2006).

- Morphometric measurements used for fasciolid adults follow a logistic growth model with respect to time (Valero et al. 2005). This implies that the morphometric development of the fasciolid adult is not limited but 'damped' and does not exceed certain characteristic maxima (Valero et al. 2001a, b, 2006b, 2011). Since the morphometric maximum values are characteristic for each population, they are considered the comparative base of this study (Table II).

\section{Data analysis}

The fasciolid adult stage undergoes a marked developmental process in the definitive host. The changes in different biometrical parameters of adult flukes at different times have been previously analysed (Valero et al. 1998, 2001a, b, 2005, 2011). The corresponding growth curves were all logistic, which implies that the morphometric development of the adult fluke is not unlimited but "damped" and cannot exceed certain characteristic maxima of ym (= maximum value attained by a biometric variable). This model describes the variation in adult fluke dimensions along time, from parasite migration to the adult location in the bile duct. Entry into the bile duct induces maturation and egg production. The logistic model which represents body growth and development is characterised by two phases (Valero et al. 1998, 2005). The "exponential" part of logistic growth corresponds to body development during migration in the abdominal cavity and liver parenchyma, as well as to development and sexual mat- 


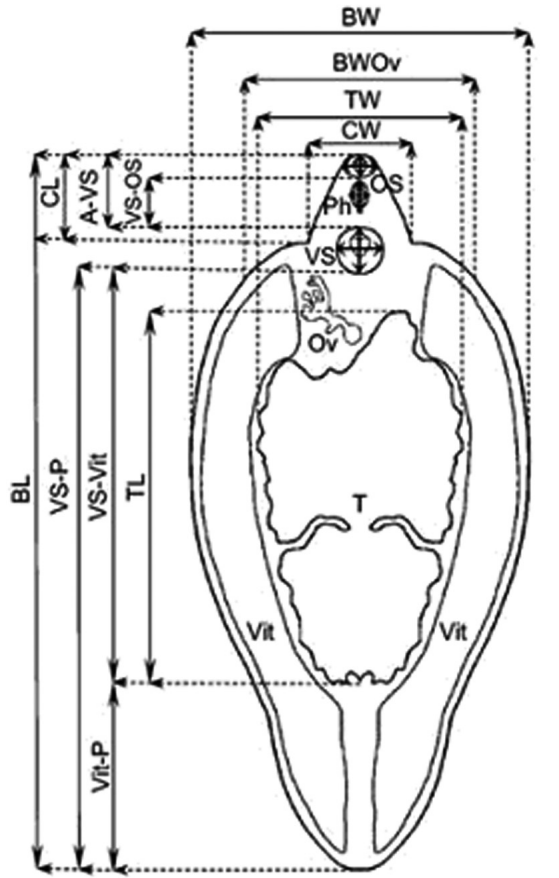

A)

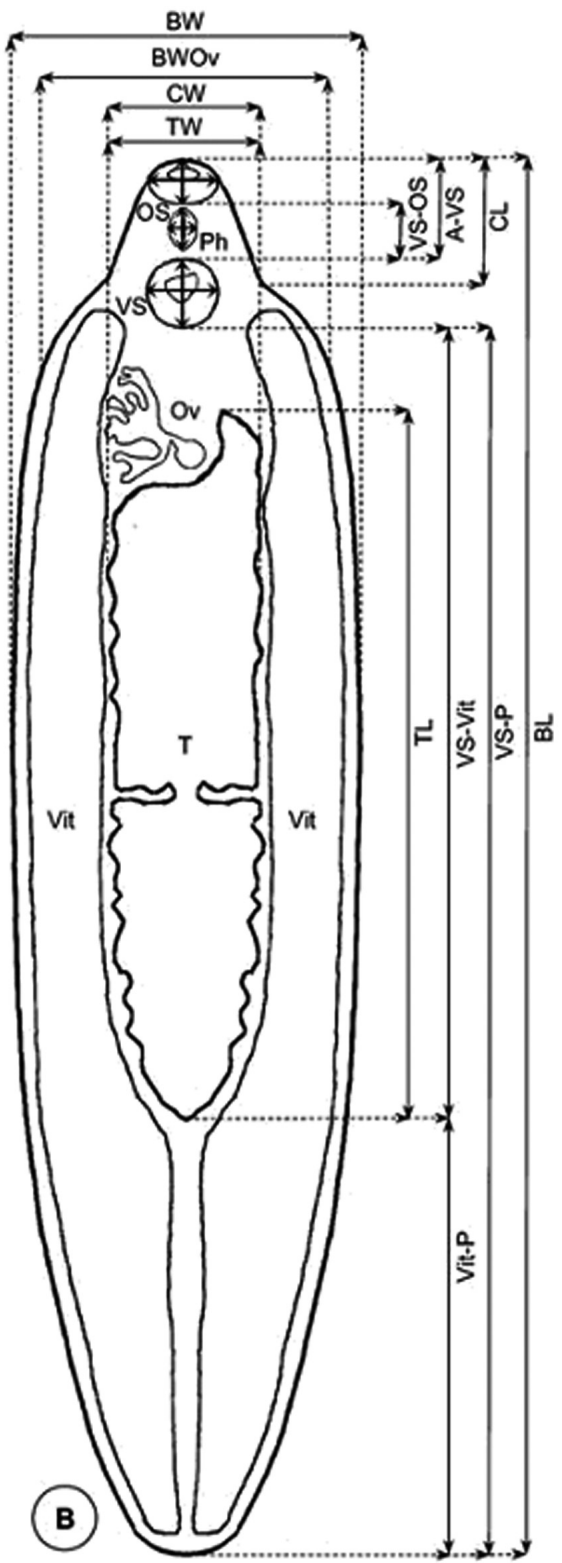

Fig.2. Standardised measurements applied for the morphometric characterisation of fasciolid adult specimens (see text for abbreviations). A - Fasciola hepatica-like forms; $\mathbf{B}$ - Fasciola gigantica-like forms

uration in the biliary duct system up to the onset of egg production.

Consequently, to avoid the influence of age and growth on the trematode comparison, morphological variation is quantified by geometrical morphometrics (Rohlf and Marcus, 1993), a technique offering an estimate of size by which different axes of growth are integrated into a single variable (Afshan et al. 2013). The estimate of size is contained in a single variable reflecting variation in many directions, as many as there are landmarks under study, and shape is defined as their relative positions after correction for size, position and orientation. With these informative data, and the corresponding software freely available to conduct complex analyses, sig- nificant biological and epidemiological features can be quantified more accurately (Dujardin, 2008).

Current statistical techniques in morphometrics make it possible to test the null hypothesis of conspecific populations being simply the allometric extension of each other, provided a common allometric trend is identifiable (Klingenberg, 1996; Dujardin and Le Pont, 2004). Multivariate analyses were applied to calculate the phenotypic variations among fasciolid adults, using size-free canonical discriminant analysis of the covariance of log-transformed measurements to assess phenotypic variations between the samples. These analyses are applied to exclude the effect of within-group ontogenetic variations by reducing the effect of each character on the first 


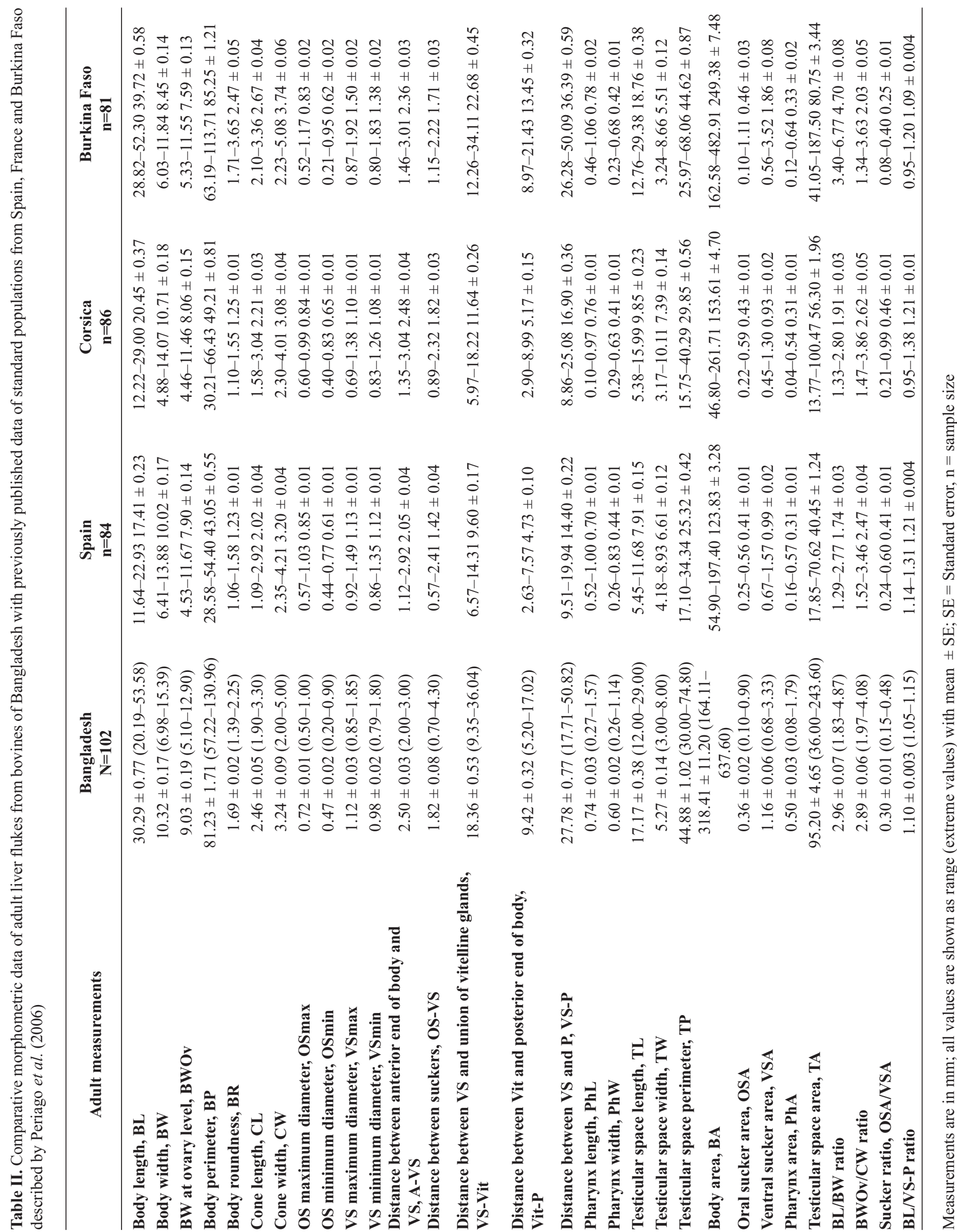




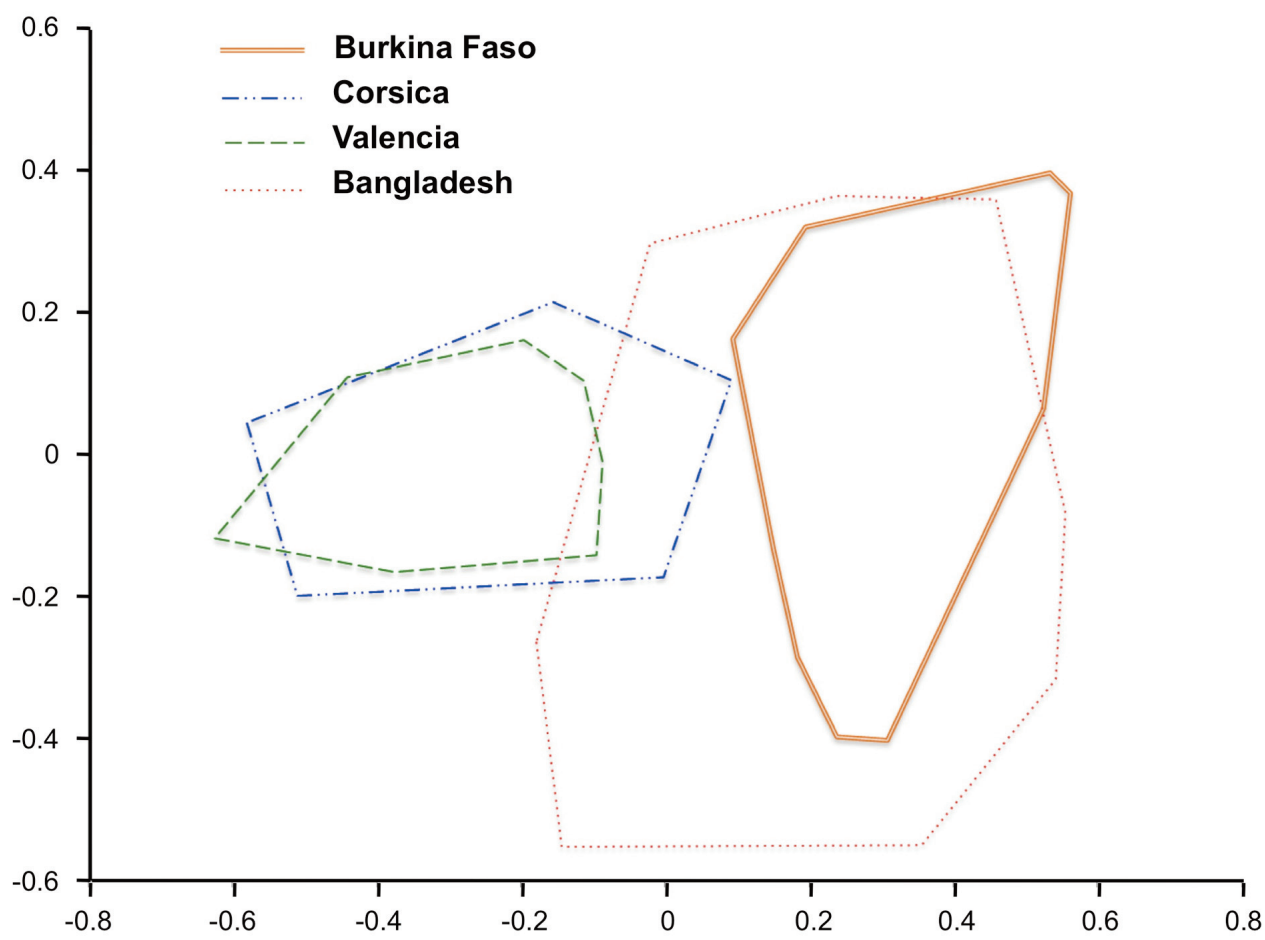

Fig. 3. Principal component analysis of adult fasciolids from natural infections in bovines of Bangladesh (dotted line) compared with F. hepatica from Valencia, Spain (dashed line) and Corsica, France (dashed-dotted line) and F. gigantica from Burkina Faso (solid line) (Periago et al. 2006). Samples are projected on to the first (PCI, 58\%) and second (PCII, 29\%) principal components

pooled within-group principal component (a multivariate size estimator) (Dos-Reis et al. 1990). The principal component analysis is used to summarize most of the variations in a multivariate dataset in a few dimensions (Dujardin and Le Pont, 2004).

Phenotypic analysis of fasciolid adults was conducted by using various modules of the CLIC package (by J.P. Dujardin, http://momedujardin.wordpress.com). The results were statistically significant when $\mathrm{P}<0.05$. The following non-redundant measurements (one measurement is not included in another) used for fasciolid adults were BL, BW, BP, VS-Vit, Vit-P, PL and PW, where at least one dimension was measured among the most important morphological characters. The remaining variables (PCII, Y axis) were all significantly correlated with the first principal component (PCI, $\mathrm{X}$ axis), contributing $58 \%$ to overall variations. The PCI could therefore be accepted as a general indicator of size (Afshan et al. 2013), so that the resulting factor maps (Fig. 3) can clearly illustrate global size differences in the populations analysed.

Although metacercarial infectivity does not appear to differ in isolates from different livestock species (Valero and Mas-Coma, 2000), the definitive animal host species is known to pronouncedly influence the phenotype of both adult stage and eggs of the liver fluke, mainly due to the different size of the liver duct microhabitat (Valero et al. 2001a, 2002, 2009a). For this reason, only parasites obtained from bovines were used in the principal component analysis carried out in the present study.

\section{Results}

Fasciolid populations from cattle from Bangladesh were grouped according to the maximum and minimum values of given differentiating morphological measurements previously proposed for $F$. hepatica, F. gigantica or Fasciola sp. (= intermediate forms) (Periago et al. 2006): BR: $1.06-1.58$ in $F$. hepatica, 1.71-3.65 in F. gigantica; BL/BW: $1.29-2.80$ in $F$. hepatica, 3.40-6.78 in F. gigantica; and VS-P: 8.86-25.08 $\mathrm{mm}$ in F. hepatica, 26.28-50.09 $\mathrm{mm}$ in F. gigantica.

The specimens from Bangladesh were first grouped according to BR and BL/BW, and, secondarily, according to VSP. Using these criteria, adult specimens from cattle were grouped into $F$. hepatica-like $(11.76 \%), F$. gigantica-like $(47.06 \%)$ or Fasciola sp.-like (41.18\%). Similarly, adult specimens from buffaloes were grouped into $F$. hepatica-like (5.88\%), F. gigantica-like $(39.22 \%)$ or Fasciola sp.-like $(54.90 \%)$.

The resulting factor maps clearly illustrate global size differences in the cattle and buffalo populations analysed, each group being represented by its perimeter (Fig. 3). Two independent zones can be distinguished: one zone is made up of 
samples from Spain and Corsica, while the other zone consists only of samples from Burkina Faso. These zones overlap with the samples from Bangladesh.

The multivariate analysis used to measure the changes in size of fasciolid adults from Bangladesh and compared with the above-mentioned standard populations for each pure fasciolid species showed that the size of most fasciolids from Bangladesh is situated between $F$. hepatica and $F$. gigantica standard populations. These results demonstrate that intermediate forms of fasciolids exist in Bangladesh. Nevertheless, it is worth mentioning that the samples from Bangladesh overlap with $F$. hepatica and $F$. gigantica standard populations, but in the absence of specimens of standard F. hepatica and F. gigantica from Bangladesh it is also possible that the forms identified as $F$. hepatica-like and $F$. gigantica-like could be the extreme values of the morphometric distribution of the Fasciola sp.-like specimens.

\section{Discussion}

The CIAS detection of the three phenotypic groups of $F$. hepatica-like forms (cattle: 11.76\%; buffalo: 5.88\%), F. gigantica-like forms (cattle: $47.06 \%$; buffalo: $39.22 \%$ ) or Fasciola sp.-like forms (cattle: $41.18 \%$; buffalo: $54.90 \%$ ) represent highly interesting results and pose several question marks.

The first aspect to be highlighted is the geographical and physiographical similarities between Bangladesh and the lowlands of Pakistan, where human fascioliasis has been reported recently (Qureshi et al. 2005; Qureshi and Tanveer, 2009) and where human infection by Fasciola has more recently proved to be related to climate and global changes (Afshan et al. 2014). Bangladesh presents abundant water bodies, irrigation fields and canals, making it a suitable habitat for lymnaeid snail vectors. Geographical locations, agro-ecological and agro-biological conditions, and climate change with the particular trend of increasing temperatures are enhancing the abundance, seasonality, reproduction and distribution of different species of snail borne trematodes throughout the country. Bangladesh is globally recognised as the most vulnerable country to climate change, e.g. in Bangladesh the average temperature has registered an upward trend of about $1^{\circ} \mathrm{C}$ in May and $0.5^{\circ} \mathrm{C}$ in November along the 14 year period from 1985 to 1998 (IPCC, 2007). According to general analyses, climate change is bound to have a severe impact on the transmission of this disease in Bangladesh (Mas-Coma et al. 2008, 2009b). Thus, similarity results suggest that a risk for human infection in Bangladesh should be considered. In that sense, the detection of $F$. hepatica-like forms becomes important, given the well-known capacity of $F$. hepatica to infect humans which is markedly higher than in F. gigantica. Present widespread fascioliasis with high pathogenicity in ruminants from Bangladesh is a good indicator for the disease likely to be diagnosed in humans if properly investigated. The higher proportion of $F$. gigantica-like forms in the country may suggest more severe pathogenic characteristics related to the larger size of F. gigantica if it infects humans (Girones et al. 2007; Valero et al. 2008).

The percentages of the three forms found in the bovines studied, with similar proportions in both cattle and buffaloes, and with a patent domination of $F$. gigantica-like forms and Fasciola sp.-like forms closer to $F$. gigantica than to $F$. hepatica, agree with the known evolutionary-historical scenario throughout southern Asia (Mas-Coma et al. 2009a). In relation to the distribution of fasciolids in Asia, historical, archeological, biogeographic, climatic and lymnaeid faunal data indicate that two different main routes from the Fertile Crescent and eastwards separated by the large Himalayan chain need to be considered (Mas-Coma et al. 2009a). One northward from the Himalaya includes the spread of mainly $F$. hepatica and another route southward from the Himalaya concerns the spread of mainly F. gigantica through the southern Asian region. This southward spread appears to have been linked to the higher temperatures and presence of the appropriate Radix vector species in the lowlands of Afghanistan, Pakistan, India and eastward up to South East Asia. This spread should have been facilitated by the extensive trade between the two primary centres of India and the Fertile Crescent during the 4000-1000 BC period and the later, very intense and long-distance commercial exchanges between those southern Asian countries and Near East countries, for instance, through the southern routes of the "Silk Road", which was active over 15 centuries, from around 138 years BC until the 15 th century. Camels, taurine and zebu cattle were mainly used for the transportation of goods and merchandise, while dromedaries were later incorporated into the most southern routes of the Silk Road through Afganistan, Pakistan and India because of their better adaptation to warmer climates (MasComa et al. 2009a).

The detection of $F$. hepatica-like forms poses the question mark about the lymnaeid vectors which may be needed for their transmission in Bangladesh. Fasciola hepatica is mainly transmitted by small size lymnaeid species of the Galba/Fossaria group (Bargues et al. 2007, 2011a), including Galba truncatula as the main vector and the only one in Europe, but also present in Asia, Africa and South America. Fasciola gigantica is transmitted by lymnaeids of larger size of the Radix group (Bargues and Mas-Coma, 2005). The presence of lymnaeid vectors defines not only the distribution of fascioliasis, but may also explain the distribution of human infection within a country, as has been recently observed in different countries (Artigas et al. 2011; Bargues et al. 2011b, c), and within an endemic area, as well as its seasonality or permanent transmission (Bargues et al. 2012). In southern Asia, G. truncatula is restricted to the highlands in countries such as Afghanistan and Pakistan (Kendall, 1954, 1965) and its absence as well as the lack of appropriate Galba/Fossaria lymnaeid species for the development of $F$. hepatica in India and eastward up to South-east Asia is well known (Mas-Coma et al. 2009a). In Bangladesh, G. truncatula has never been 
found, nor does the country have temperatures below $15^{\circ} \mathrm{C}$ (except for about 10 days a year), which indicates a scenario not appropriate for the transmission of $F$. hepatica. Consequently, it may be perhaps better to consider that the forms identified as $F$. hepatica-like could indeed correspond to the extreme values of the morphometric distribution of the Fasciola sp.-like specimens.

Previous studies have shown the presence of morphologically intermediate forms in geographically sympatric areas of F. hepatica and F. gigantica in Africa and Asia. In Africa, the presence of intermediate forms has been morphometrically evidenced by means of the CIAS methodology in Egypt (Periago et al. 2008). In Asia, a varied spectrum of morphological forms of fasciolids has been described based on traditional microscopic measurements in several countries, such as India (Varma, 1953), Japan (Watanabe, 1962; Terasaki et al. 2000), Korea (Chu and Kim, 1967), the Philippines (Kimura et al. 1984) and Thailand (Srimuzipo et al. 2000), and more recently by CIAS also in Iran (Moghaddam et al. 2004; Ashrafi et al. 2006, 2015) and Pakistan (Asfhan et al. 2013).

The present phenotypic study by the CIAS method on fasciolids infecting bovines demonstrates, for the first time in Bangladesh, the presence of intermediate forms (here noted as Fasciola sp.-like forms) and $F$. hepatica-like forms, besides the $F$. gigantica-like forms always reported in the country as simply F. gigantica. Interestingly, and agreeing with these results, fascioliasis caused by a shorter strain of $F$. gigantica has been sporadically described in Bangladesh (Robertson, 1976; Nooruddin and Islam, 1996). An additional study of liver flukes from livestock by means of PCR-RFLP of nuclear rDNA ITS-1 and short fragment (535 bp) sequences of the mtDNA nad 1 also detected a heterogeneity in the liver flukes from that country, when finding that (i) 29 out of 127 aspermic flukes presented a combination of ITS-1 sequences of Fasciola gigantica and Fasciola hepatica, and (ii) all flukes presented nad 1 sequences identical to aspermic Fasciola sp. from other Asian countries (Mohanta et al. 2014)

The increasing recent prevalences found in livestock, the proved impact of climate change on Asian latitudes such as those of Bangladesh, and the risk for human infection due to the aforementioned climate change effects on the disease transmission as already reported in the similar situation in Pakistan, suggest a worrying scenario. Further studies on the transmission and epidemiology of the disease in humans as well as animals are already under way to obtain the baseline on which to establish appropriate control measures against fascioliasis in Bangladesh.

Acknowledgements. Funds were provided by the Bangladesh Academy of Science-USDA LS-07 projects for research in the Department of Pathology, Faculty of Veterinary Science, Bangladesh Agricultural University, Mymensingh, Bangladesh and the Contingency Project for Institutional Development (Oracle Project Nos. 2060995 and RAS0066) of the International Atomic Energy Agency (IAEA). The present study was performed during the stay of the first author in the
Department of Parasitology, Faculty of Pharmacy, University of Valencia, Spain, funded by a fellowship of the IAEA (Headquarters Vienna, Code No. BGD/13034).

Spanish collaboration funded by Projects No. SAF2010-20805 of the Ministry of Economy and Competitiveness, Madrid, Spain; by Project No. RD12/0018/0013, Red de Investigación Cooperativa en Enfermedades Tropicales - RICET, IV National Program of I+D+I 2008-2011, ISCIII - Subdirección General de Redes y Centros de Investigación Cooperativa and FEDER, Ministry of Health and Consumption, Madrid, Spain; and by Project No. 2012/042 of the PROMETEO Program, Generalitat Valenciana, Valencia, Spain.

This study is part of the worldwide initiative of WHO (Headquarters Geneva, Switzerland) against human fascioliasis.

\section{References}

Afshan K., Valero M.A., Qayyum M., Peixoto R.V., Magraner A., Mas-Coma S. 2013. Phenotypes of intermediate forms of Fasciola hepatica and F. gigantica in buffaloes from Central Punjab, Journal of Helminthology, 88, 417-426. DOI: 10.1017/S0022149X13000369

Afshan K., Fortes-Lima C.A., Artigas P., Valero M.A., Qayyum M., Mas-Coma S. 2014. Impact of climate change and man-made irrigation systems on the transmission risk, long-term trend and seasonality of human and animal fascioliasis in Pakistan. Geospatial Health, 8, 317-334

Artigas P., Bargues M.D., Mera y Sierra R., Agramunt V.H., MasComa S. 2011. Characterisation of fascioliasis lymnaeid intermediate hosts from Chile by DNA sequencing, with emphasis on Lymnaea viator and Galba truncatula. Acta Tropica, 120, 245-257. DOI: 10.1016/j.actatropica.2011.09.002

Ashrafi K., Valero M.A., Panova M., Periago M.V., Massoud J., MasComa S. 2006. Phenotypic analysis of adults of Fasciola hepatica, Fasciola gigantica and intermediate forms from the endemic region of Guilan, Iran. Parasitology International, $55,249-260$

Ashrafi K., Valero M.A., Peixoto R.V., Artigas P., Panova M., Mas-Coma S. 2015. Distribution of Fasciola hepatica and F. gigantica in the endemic area of Guilan, Iran: Relationships between zonal overlap and phenotypic traits. Infection, Genetics and Evolution, 31, 95-109. DOI: 10.1016/j.meegid.2015.01.009

Bangladesh Academy of Science (BAS)-USDA, 2012. Annual Project Report of LS 07/2011 project, Mondal MMH, Bangladesh Agricultural University, Mymensingh

Bargues M.D., Mas-Coma S. 2005. Reviewing lymnaeid vectors of fascioliasis by ribosomal DNA sequence analyses. Journal of Helminthology, 79, 257-267

Bargues M.D., Artigas P., Mera y Sierra R.L., Pointier J.P., MasComa S., 2007. Characterisation of Lymnaea cubensis, L. viatrix and $L$. neotropica $\mathrm{n}$. sp., the main vectors of Fasciola hepatica in Latin America, by analysis of their ribosomal and mitochondrial DNA. Annals of Tropical Medicine and Parasitology, 101, 621-641

Bargues M.D., Artigas P., Khoubbane M., Flores R., Glöer P., Rojas-García R., Ashrafi K., Falkner G., Mas-Coma S. 2011 a. Lymnaea schirazensis, an overlooked snail distorting fascioliasis data: genotype, phenotype, ecology, worldwide spread, susceptibility, applicability. PLoS ONE, 6, e24567 (33 pp. + 3 Suppl. Tables +5 Suppl. Figures). DOI: 10.1371/journal.pone. 0024567

Bargues M.D., Gonzalez C., Artigas P., Mas-Coma S. 2011b. A new baseline for fascioliasis in Venezuela: lymnaeid vectors ascertained by DNA sequencing and analysis of their relation- 
ships with human and animal infection. Parasites \& Vectors, 4, 200 (18 pp.). DOI: 10.1186/1756-3305-4-200

Bargues M.D., Artigas P., Khoubbane M., Mas-Coma S. 2011c. DNA sequence characterisation and phylogeography of Lymnaea cousini and related species, vectors of fascioliasis in northern Andean countries, with description of L. meridensis $\mathrm{n}$. sp. (Gastropoda: Lymnaeidae). Parasites \& Vectors, 4, 132 (22 pp.). DOI: 10.1186/1756-3305-4-132

Bargues M.D., Artigas P., Khoubbane M., Ortiz P., Naquira C., MasComa S. 2012. Molecular characterisation of Galba truncatula, Lymnaea neotropica and L. schirazensis from Cajamarca, Peru and their potential role in transmission of human and animal fascioliasis. Parasites \& Vectors, 5, 174 (16 pp.). DOI: 10.1186/1756-3305-5-174

Bhuiyan M.A. 1970. A survey of helminth parasitizing the liver of domesticated ruminants in East Pakistan. M.Sc. Thesis, Department of Parasitology, Faculty of Veterinary Science, Bangladesh Agricultural University (Former EPAU), Mymensingh

Chen M.G., Mott K.E., 1990. Progress in assessment of morbidity due to Fasciola hepatica infection: a review of recent literature. Tropical Diseases Bulletin, 87, R1-R38

Chu J.K., Kim Y.K. 1967. Taxonomical study on the Fasciolidae in Korea. Korean Journal of Parasitology, 5, 139-146

Dos-Reis S.P., Pessoa L.M., Strauss R.E. 1990. Application of sizefree canonical discriminant analysis to studies of geographic differentiation. Brazilian Journal of Genetics, 13, 509-520

Dujardin J.P. 2008. Morphometrics applied to medical entomology. Infection, Genetics and Evolution, 8, 875-890

Dujardin J.P., Le-Pont F. 2004. Geographical variation of metric properties within the neotropical sandflies. Infection, Genetics and Evolution, 4, 353-359

Fuentes M.V., Valero M.A., Bargues M.D., Esteban J.G., Angles R., Mas-Coma S. 1999. Analysis of climatic data and forecast indices for human fascioliasis at very high altitude. Annals of Tropical Medicine and Parasitology, 93, 835-850

Fuentes M.V., Malone J.B., Mas-Coma S. 2001. Validation of a mapping and predicting model for human fasciolosis transmission in Andean very high altitude endemic areas using remote sensing data. Acta Tropica, 79, 87-95

Girones N., Valero M.A., García-Bodelón M.A., Chico-Calero M.I., Punzón C., Fresno M., Mas-Coma S. 2007. Immune supression in advanced chronic fascioliasis: an experimental study in a rat model. Journal of infectious Diseases, 195, 1504-1512

Hossain M.M., Paul S., Rahman M.M., Hossain F.M.A., Hossain M.T., Islam M.R. 2011. Prevalence and economic significance of caprine fascioliasis at Sylhet district of Bangladesh. Pakistan Veterinary Journal, 3, 113-116

Ichikawa M., Bawn S., Mawd N.N., Htun L.L., Thein M., Gyi A., Sunn K., Katakura K., Itagaki T. 2011. Characterization of Fasciola spp. in Myanmar on the basis of spermatogenesis status and nuclear and mitochondrial DNA markers. Parasitology International, 60, 474-479. DOI: 10.1016/j.parint. 2011.08.007

IPCC. 2007. Intergovernmental Panel on Climate Change. Climate Change: Synthesis Report; Summary for Policymakers. Working Group contributions to the Fourth Assessment Report, IPCC Plenary XXVII (Valencia, Spain, 12-17 November 2007). Retrieved from: http://www.ipcc.ch/pdf/assessmentreport/ar4/syr/ar4 syr_spm.pdf

Kendall S.B. 1954. Fascioliasis in Pakistan. Annals of Tropical Medicine and Parasitology, 43, 307-313

Kendall S.B. 1965. Relationships between the species of Fasciola and the molluscan hosts. Advances in Parasitology, 3, 59-98

Kimura S., Shimizu A., Kawano J. 1984. Morphological observation on liver fluke detected from naturally infected carabaos in the
Philippines. The Science Reports of the Faculty of Agriculture, Kobe University, 16, 353-357

Klingenberg C.P. 1996. Multivariate allometry. In: (Eds. L.F. Marcus, M. Corti, A. Loy, G.J.P. Naylor, D. Slice) Advances in Morphometrics. Proceedings of the 1993 NATO-ASI on Morphometrics, NATO ASI, Ser. A, Life Sciences. Plenum Publishers, New York, 23-49

Mas-Coma S., Valero M.A., Bargues M.D. 2008. Effects of climate change on animal and zoonotic helminthiases. Revue Scientifique et Technique de l'Office Internationale des Epizooties, 27, 443-457

Mas-Coma S., Valero M.A., Bargues M.D. 2009a. Fasciola, lymnaeids and human fascioliasis, with a global overview on disease transmission, epidemiology, evolutionary genetics, molecular epidemiology and control. Advances in Parasitology, 69, 41-146. DOI: 10.1016/S0065-308X(09)69002-3

Mas-Coma, S., Valero, M.A., Bargues, M.D., 2009b. Climate change effects on trematodiases, with emphasis on zoonotic fascioliasis and schistosomiasis. Veterinary Parasitology, 163, 264 280. DOI: 10.1016/j.vetpar.2009.03.024

Mas-Coma S., Agramunt V.H., Valero M.A. 2014a. Neurological and ocular fascioliasis in humans. Advances in Parasitology, 84, 27-149. DOI: 10.1016/B978-0-12-800099-1.00002-8

Mas-Coma S., Bargues M.D., Valero M.A. 2014b. Diagnosis of human fascioliasis by stool and blood techniques: Update for the present global scenario. Parasitology, 141, 1918-1946. DOI: $10.1017 / \mathrm{S} 0031182014000869$

Moghaddam A.S., Massoud J., Mahmoodi M., Mahvi A.H., Periago M.V., Artigas P., Fuentes M.V., Bargues M.D., Mas-Coma S. 2004. Human and animal fascioliasis in Mazandaran province, northern Iran. Parasitology Research, 94, 61-69

Mohanta U.K., Ichikawa-Seki M., Shoriki T., Katakura K., Itagaki T. 2014. Characteristics and molecular phylogeny of Fasciola flukes from Bangladesh, determined based on spermatogenesis and nuclear and mitochondrial DNA analyses. Parasitology Research, 113, 2493-2501. DOI: 10.1007/ s00436-014-3898-5

Nooruddin M., Islam K.S. 1996. Distribution and body size of Fasciola gigantica in livers of Bengal goats in Bangladesh. Small Ruminant Research, 19, 189-191. DOI: None.

Periago M.V., Valero M.A., Panova M., Mas-Coma S. 2006. Phenotypic comparison of allopatric populations of Fasciola hepatica and Fasciola gigantica from European and African bovines using a computer image analysis system (CIAS). $\mathrm{Pa}$ rasitology Research, 99, 368-378

Periago M.V., Valero M.A., El-Sayed M., Ashrafi K., El-Wakeel A., Mohamed M.Y., Desquesnes M., Curtale F., Mas-Coma S. 2008. First phenotypic description of Fasciola hepatica/Fasciola gigantica intermediate forms from the human endemic area of the Nile Delta, Egypt. Infection, Genetics and Evolution, 8, 51-58

Qureshi A.W., Tanveer A. 2009. Seroprevalence of fasciolosis in buffaloes and humans in some areas of Punjab, Pakistan. Pakistan Journal of Science, 61, 91-96

Qureshi A.W., Tanveer A., Qureshi S.W., Maqbool A., Gill T.J., Ali S.A. 2005. Epidemiology of human fasciolosis in rural areas of Lahore, Pakistan. Punjab University Journal of Zoology, 20, 159-168

Robertson A. 1976. Handbook on Animal Diseases in the Tropics. 3rd Edition. British Veterinary Association, 7 Mansfield Street, London W 1 M OAT, UK, pp. 227-230

Rohlf F.J., Marcus L.F. 1993. A revolution in morphometrics. Trends in Ecology and Evolution, 8, 129-132. DOI: 10.1016/01695347(93)90024-J

Spithill T.W., Smooker P.M., Copeman D.B. 1999. Fasciola gigantica: epidemiology, control, immunology and molecular biol- 
ogy. In: (Ed. J.P. Dalton) Fasciolosis, CAB International Publishing, Wallingford, 465-525

Srimuzipo P., Komalamisra C., Choochote W., Jitpakdi A., Vanichthanakorn P., Keha P., Riyong D., Sukontasan K., Komalamisra N., Sukontasan K., Tippawangkosol P. 2000. Comparative morphometry, morphology of egg and adult surface topography under light and scanning electron microscopies, and metaphase karyotype among three size-races of Fasciola gigantica in Thailand. Southeast Asian Journal of Tropical Medicine and Public Health, 31, 366-373

Terasaki K., Noda Y., Shibahara T., Itagaki T. 2000. Morphological comparisons and hypotheses on the origin of polyploids in parthenogenetic Fasciola sp. Journal of Parasitology, 86, 724-729

Torgerson P., Claxton J. 1999. Epidemiology and control. In: (Ed. J.P. Dalton) Fasciolosis, CAB International Publishing, Wallingford, 113-149

Valero M.A, Mas-Coma S. 2000. Comparative infectivity of Fasciola hepatica metacercariae from isolates of the main and secondary reservoir animal host species in the Bolivian Altiplano high human endemic region. Folia Parasitologica, 47, 17-22

Valero M.A., Marcos M.D., Fons R., Mas-Coma S. 1998. Fasciola hepatica development in experimentally infected black rat, Rattus rattus. Parasitology Research, 84, 188-194

Valero M.A., Darce N.A., Panova M., Mas-Coma S. 2001a. Relationships between host species and morphometric patterns in Fasciola hepatica adults and eggs from the Northern Bolivian Altiplano hyperendemic region. Veterinary Parasitology, 102, 85-100

Valero M.A., Panova M., Mas-Coma S. 2001b. Development differences in the uterus of Fasciola hepatica between livestock liver fluke populations from Bolivian highland and European lowlands. Parasitology Research, 87, 337-342

Valero M.A., Panova M., Comes A.M., Fons R., Mas-Coma S. 2002. Patterns in size and shedding of Fasciola hepatica eggs by naturally and experimentally infected murid rodents. Journal of Parasitology, 88, 308-313

Valero M.A., Santana M., Morales M., Hernandez J.L., Mas-Coma, S. 2003. Risk of gallstone disease in advanced chronic phase of fascioliasis: an experimental study in a rat model. Journal of Infectious Diseases, 188, 787-793

Valero M.A., Panova M., Mas-Coma S. 2005. Phenotypic analysis of adults and eggs of Fasciola hepatica by computer image analysis system. Journal of Helminthology, 79, 217-225

Valero M.A., Navarro M., Garcia-Bodelon M.A., Marcilla A., Morales M., Garcia J.E., Hernandez J.L., Mas-Coma S. 2006a. High risk of bacterobilia in advanced experimental chronic fasciolosis. Acta Tropica, 100, 17-23

Valero M.A., De Renzi M., Panova M., García-Bodelón MA, Periago M.V., Ordoñez D., Mas-Coma S. 2006b. Crowding effect on adult growth, pre-patent period and egg shedding of Fasciola hepatica. Parasitology. 133, 453-463

Received: May 19, 2015

Revised: September 11, 2015

Accepted for publication: December 1, 2015
Valero M.A., Girones N., Garcia-Bodelon M.A., Periago M.V., Chico-Calero I., Khoubbane M., Fresno M., Mas-Coma S., 2008. Anaemia in advanced chronic fasciolosis. Acta Tropica, $108,35-43$

Valero M.A., Perez-Crespo I., Periago M.V., Khoubbane M., MasComa S. 2009a. Fluke egg characteristics for the diagnosis of human and animal fascioliasis by Fasciola hepatica and $F$. gigantica. Acta Tropica, 111, 150-159. DOI: 10.1016/j.actatropica.2009.04.005

Valero M.A., Ubeira F.M., Khoubbane M., Artigas P., Muiño L., Mezo M., Pérez-Crespo I., Periago M.V., Mas-Coma S. 2009b. MM3-ELISA evaluation of coproantigen release and serum antibody production in sheep experimentally infected with Fasciola hepatica and F. gigantica. Veterinary Parasitology, 159, 77-81. DOI: 10.1016/j.vetpar.2008.10.014

Valero M.A., Panova M., Pérez-Crespo I., Khoubbane M., MasComa S. 2011. Correlation between egg-shedding and uterus development in Fasciola hepatica human and animal isolates: applied implications. Veterinary Parasitology, 183, 79-86. DOI: 10.1016/j.vetpar.2011.07.003

Valero M.A., Periago M.V., Pérez-Crespo I., Rodríguez E., Perteguer M.J., Garate T., González-Barbera E.M., Mas-Coma S. 2012a. Assessing the validity of an ELISA test for the serological diagnosis of human fascioliasis in different epidemiological situations. Tropical Medicine and International Health, 17, 630-636. DOI: 10.1111/j.1365-3156.2012.02964.x

Valero M.A., Periago M.V., Pérez-Crespo I., Angles R., Villegas F., Aguirre C., Strauss W., Espinoza J.R., Herrera P., Terashima A., Tamayo H., Engels D., Gabrielli A.F., Mas-Coma S. $2012 \mathrm{~b}$. Field evaluation of a coproantigen detection test for fascioliasis diagnosis and surveillance in human hyperendemic areas of Andean countries. PLoS Neglected Tropical Diseases, 6, e1812. DOI: 10.1371/journal.pntd.0001812

Valero M.A., Perez-Crespo I., Khoubbane M., Artigas P., Panova M., Ortiz P., Maco V., Espinoza J.R., Mas-Coma S. 2012c. Fasciola hepatica phenotypic characterization in Andean human endemic areas: valley versus altiplanic patterns analysed in liver flukes from sheep from Cajamarca and Mantaro, Peru. Infection, Genetics and Evolution, 12, 403-410. DOI: 10.1016/j.meegid.2012.01.009

Varma A.K. 1953. On Fasciola indica n. sp. with some observations on F. hepatica and F. gigantica. Journal of Helminthology, 27, 185-198

Watanabe S. 1962. Fasciolosis of ruminants in Japan. Bulletin de l'Office International des Epizooties, 58, 313-322

World Health Organization. 2013. Sustaining the drive to overcome the global impact of neglected tropical diseases. World Health Organization, WHO Headquarters, Geneva, pp. 138 